\title{
Gravity and Geometric Phases
}

\author{
A. Corichi and M. Pierri \\ Center for Gravitational Physics and Geometry \\ Department of Physics, The Pennsylvania State University \\ University Park, PA 16802 \\ December 1st, 1994
}

\begin{abstract}
The behavior of a quantum test particle satisfying the Klein-Gordon equation in a certain class of 4 dimensional stationary space-times is examined. In a space-time of a spinning cosmic string, the wave function of a particle in a box is shown to acquire a geometric phase when the box is transported around a closed path surrounding the string. When interpreted as an Aharonov-Anandan geometric phase, the effect is shown to be related to the Aharonov-Bohm effect.
\end{abstract}

\section{Introduction}

The term "gravitational Aharonov-Bohm effect" (gAB) has been used to describe a variety of systems with diverse properties [1, 2]. Usually it implies a behavior different from the one in Minkowski space-time even when the motion is restricted to regions in which the space-time is (locally) flat. At the classical level, the parallel transport of vectors and spinors round a cosmic string, for example, is nontrivial when the mass $M$ of the string is different from zero. When the angular momentum $J$ is non vanishing, the time required for null (light) rays to travel around the cosmic string depends on the direction of motion [3]. For a quantum test (Klein Gordon) particle the effect is manifested in different ways depending on the situation. When bound states are studied [2] it is seen that the energy spectrum is modified depending on the mass and angular momentum of the string. In the scattering of particles, the scattering amplitude is non trivial and the phase shifts are modified also depending on $M$ and $J$ [4].

It has also been noted that in linearized gravity, i.e. with $g_{a b}=\eta_{a b}+h_{a b}$ where $h_{a b}$ is small, the deparametrized square-root Hamiltonian of a slowly moving particle takes the form: $H=\left(p_{i}-A_{i}\right)^{2}+\frac{1}{2} m h_{00}$ where $A_{i}=\frac{1}{2} h_{0 i} . A_{i}$ transforms like a $U(1)$ connection under infinitesimal diffeomorphism $x^{i} \rightarrow x^{i}+\zeta^{i}$ and if $\partial_{[i} A_{j]}=0$, the Schrödinger equation looks like the one in the $\mathrm{AB}$ case, $h_{0 i}$ playing the role of 
the electromagnetic potential [5]. Using this analogy, Anandan obtained a geometric phase for a particle satisfying the Schrödinger equation in the weak field region around a cosmic string by interfering beams passing on opposite sides of the string. The wave function thus constructed is, however, not single-valued [6].

We will show that this last construction can be extended to general metrics (without the weak field assumption) and for the fully relativistic Klein-Gordon equation (so that the velocity is not necessarily small) using only single-valued solutions. The $g_{0 i}$ part of the metric still behaves like a potential. Both the Hamiltonian constraint and the Klein Gordon equation have a very appealing form in terms of a fiducial static metric on which the potential $A_{i}$ "lives". This will allow us to identify $g_{0 i}$ as a vector potential and, in the case of locally flat space-times (which include cosmic strings ), discuss a fully relativistic gAB effect.

The similarities between charged quantum particles in the presence of a long thin solenoid and scalar quantum particles in the space-time generated by a cosmic string, are at two levels: 1) The Schrödinger equation for the charged particle and the KleinGordon equation have a similar form ; 2) There exists a "vector potential" in the linearized case that behaves like a electromagnetic $U(1)$ connection. Nevertheless, it is not apriori clear to what extent $g_{0 i}$ can be taken as a genuine connection and what its role is in the gAB effect, as compared to the role of $A_{i}$ in the electromagnetic $\mathrm{AB}$ effect. The aim of this work is to try to clarify this point.

The structure of the paper is as follows. In section 2, we consider the Klein-Gordon equation for a specific class of stationary space-times that can be decomposed into a fiducial static background metric plus a "potential $A_{i}$ term". We show that the solutions can be constructed from solutions on the "background" metric using a prescription introduced by Dirac. For the specific case of the space-time of a spinning cosmic string further properties hint at a gAB effect. In section 3 we review the electromagnetic Aharonov-Bohm effect. Following Berry's suggestion, it can be interpreted as an Aharonov-Anandan geometric phase i.e. the holonomy of a natural connection in the Hilbert space of states. Section 4 recalls that, for a stationary space-time, the space of solutions of the Klein-Gordon equation can be given the structure of a complex Hermitian Hilbert space. In section 5, we combine the results of sections 2, 3 and 4 to conclude that a geometric phase does exist for the spinning cosmic string. The phase is explicitly constructed. It provides a fully relativistic gravitational Aharonov-Bohm effect.

Throughout the paper $G=\hbar=c=1$ units are assumed.

\section{Test Particle on Stationary Space-times}

Stationary solutions of vacuum Einstein equations can describe the space-time geometry outside rotating bodies. As is well known such metrics are characterized by having a timelike Killing vector field $t^{a}$. Let us consider the following stationary 
line-element [7]:

$$
d s^{2}=-V^{2}\left(d t-A_{i} d x^{i}\right)^{2}+h_{i j} d x^{i} d x^{j}
$$

where $V, A_{i}, h_{i j}$ are functions on a Cauchy surface $\Sigma$ coordinatized by $x^{i}, i=1,2,3$. $A_{i}$ has vanishing curl $\partial_{[i} A_{j]}=0$. The time coordinate $t$ is the affine parameter along the timelike Killing vector field $t^{a}$. The freedom left in the the choice of $t$ is $t \longrightarrow t+$ $\Lambda\left(x^{i}\right)$ whereas $x^{i}$ can be transformed among themselves completely arbitrarily without changing the general form of the metric. Under this coordinate transformation, the object $A_{i}$ transforms as $A_{i} \longrightarrow A_{i}+\nabla_{i} \Lambda$. Under diffeomorphisms of the 3 manifold $\Sigma, A_{i}$ transforms as a covector. We can therefore interpret $A_{i}$ as a $U(1)$ connection under gauge transformations, living on the fiducial static space-time with metric $\stackrel{\circ}{g}_{a b}=-V^{2} \nabla_{a} t \nabla_{b} t+h_{i j} \nabla_{a} x^{i} \nabla_{b} x^{j}$

Assume that we have massive scalar quantum test particles whose behavior is determined by the Klein-Gordon equation,

$$
\left(\square-m^{2}\right) \Psi=0 .
$$

For the line element (11) it takes the form,

$$
\begin{aligned}
\left\{\stackrel{\stackrel{\rho}{ }+h^{i j} A_{i} A_{j} \partial_{0}{ }^{2}}{ }\right. & +2 h^{i j} A_{j} \partial_{0} \partial_{i}+ \\
& \left.+\frac{1}{V \sqrt{h}} \partial_{i}\left(h^{i j} A_{j} V \sqrt{h} \partial_{0}\right)-m^{2}\right\} \Psi=0,
\end{aligned}
$$

where $\stackrel{\circ}{\square}$ is the operator corresponding to $\stackrel{\circ}{g}_{a b}, h^{i j}$ is the inverse of $h_{i j}$ and $h=\operatorname{det}\left(h_{i j}\right)$. Since the background is time independent, we can look for solutions of the form

$$
\Psi(t, x)=e^{-i E t} \Phi(x)
$$

Substituting (4) into (3) we obtain an equation for $\Phi(x)$. It is a curious fact that its solution can be written in terms of the solution $\Phi_{0}(x)$ to the Klein-Gordon equation $\left(\stackrel{\circ}{\square}-m^{2}\right) \Phi_{0}=0$ on the background metric $\stackrel{\circ}{g}_{a b}$,

$$
\Phi(x)=e^{i E \int_{\mathbf{x}_{0}}^{\mathbf{x}} A_{i} d x^{i}} \Phi_{0}(x)
$$

This is the Dirac phase factor method. Although $\Psi(t, x)$ is a solution, it is multivalued and therefore should be treated with care as we will see in section 5 . For a non-contractible closed path $C$ the multi-valued feature of solution (4) is transparent.

One remark is in order. The requirement that $\partial_{[i} A_{j]}=0$ means that, locally, the connection $A_{i}$ is pure gauge, i.e. that locally the metric (1) can be put in a static form. More precisely, it implies that the twist of the Killing field $t^{a}$ is zero and therefore the nonintegrabillity of the hypersurfaces has a topological origin円, namely $H^{1}(\Sigma) \neq 0$.

\footnotetext{
${ }^{1}$ If we regard $F_{a b}=\nabla_{a} t_{b}$ as an "Electromagnetic two form", its magnetic field (proportional to the twist) would be zero; formally the situation is the same as in the electromagnetic AB case.
} 
A particular case of (11) is the space-time generated by a spinning cosmic string, on which we will focus from now on. In cylindrical coordinates $(t, \rho, \theta, z)$ the line element is given by (1) with

$$
\begin{aligned}
V^{2} & =1 \\
A_{i} & =-4 J \nabla_{i} \theta \\
h_{i j} & =\nabla_{i} \rho \nabla_{j} \rho+(\alpha \rho)^{2} \nabla_{i} \theta \nabla_{j} \theta+\nabla_{i} z \nabla_{j} z,
\end{aligned}
$$

where $J$ is the angular momentum of the string and $\alpha=1-4 \mu$ with $\mu$ the linear mass density. This space-time is flat (outside the origin) and can be locally written as the Minkowski line element. As a consequence of non vanishing $\mu$ the spatial slices $(\mathrm{t}=$ constant $)$ are planes with a wedge of angle $\beta=8 \pi \mu$ removed and edges identified, i.e., a cone. For a non vanishing $J$, the hypersurfaces which are locally orthogonal to the timelike Killing vector field, can not be globally integrated.

If one takes the Killing vector field $\left(\frac{\partial}{\partial z}\right)^{a}$ and reduces along its orbits, one gets a $2+1$ space-time with a massive spinning particle at the origin. The mass of the particle is $\mu$ and its angular momentum is $J$. For a detailed description of such spacetime see [8]. Therefore there exist two equivalent descriptions, one four dimensional and the other three dimensional.

There are some surprising results related to this space-time already in classical physics that are worth pointing out. Let us examine the effects of parallel transport of spinors and vectors along non-contractible loops. Although this space-time is locally flat everywhere (except at the origin) holonomies are non trivial. The components of vectors undergo a change which is sensitive to the angular defect $\beta$ as well as the angular momentum $J$. On the other hand the components of spinors detect just the angular defect $\beta$.

Finally, let us consider quantum particles. Using (4) and (5), it follows that the solution to the Klein-Gordon equation in this background is given by

$$
\Psi(t, \rho, \theta, z)=e^{-i E t} \Phi(\rho, \theta, z)
$$

where

$$
\Phi(\rho, \theta, z)=e^{-i 4 J E \int_{\theta_{0}}^{\theta} d \theta} \Phi_{0}(\rho, \theta, z),
$$

$\Phi_{0}(x)$ being a solution on the space-time of a non rotating string. In the next section we will see how the phase-factor gives rise to some interesting results.

\section{Geometric phase and the Aharonov-Bohm effect}

The property that the solution of a "complicated" equation can be found from solutions to a "simpler" equation using the Dirac phase factor is a well known feature 
of the electromagnetic Aharonov-Bohm (AB) wave function. In that case the solution for the Schrödinger equation for a charged particle in the exterior of a solenoid containing a magnetic flux $\phi$ can be constructed from the solution with no flux. One common exposition of the $\mathrm{AB}$ effect is to consider two beams of particles passing on opposite sides of the solenoid. The wave function for each beam is constructed from the solution without magnetic flux using the Dirac phase factor along each path. The wave functions of the two beams will be related by $\exp \left(i \oint A_{i} d x^{i}\right)$, which is precisely the holonomy of the electromagnetic $U(1)$ connection around the flux source. One then argues that the $\mathrm{AB}$ effect is due to the non triviality of the holonomy even though the curvature of the connection $F_{\mu \nu}$ vanishes. There is, however, a problem with this reasoning: the wave function is not single valued. This implies that, in spite of manifest axisymmetry, the angular momentum of the particle is not conserved as one switches on the flux on the solenoid adiabatically and is therefore not acceptable [9]. Indeed, in the analysis of a scattering situation, studied by Aharonov and Bohm in detail, it is important that the wave function is required to be single valued and the effect is observed as a nontrivial scattering amplitude [10]. Thus, strictly speaking one cannot use the naive argument. Nevertheless, one can still use the Dirac phase factor and relate the $\mathrm{AB}$ effect to the holonomy of a connection following Berry's suggestion that the $\mathrm{AB}$ effect is a particular case of Berry's phase [11]. However, the experimental setup has changed in this setting: one considers a particle contained in a box and the interference occurs between two particles one of which was transported around the flux line. Berry's phase is a particular case of the Aharonov-Anandan geometric phase which we will now introduce [12].

Consider a Hilbert space $\mathcal{H}$ and the set $\mathcal{H}_{o}$ of normalized vectors, i.e.,

$$
\mathcal{H}_{o}=\{|\Psi>\in \mathcal{H} /<\Psi| \Psi>=1\} .
$$

Define an equivalence relation $\sim$ in $\mathcal{H}_{o}$ by: $|\Psi>\sim| \Phi>$ iff $\left|\Psi>=e^{i f}\right| \Phi>$ where $f$ is real. The ray space $\mathcal{P}$ is defined as the quotient space,

$$
\mathcal{P}=\mathcal{H}_{o} / \sim
$$

and represents the space of all physically distinct states. $\mathcal{H}_{o}$ has the structure of a $U(1)$ principal bundle over $\mathcal{P}$. Suppose that the system undergoes a cyclic evolution in $\mathcal{P}$ generated by a Hamiltonian $H, i \frac{d}{d t}|\Psi>=H(t)| \Psi>$. Denote by $C$ the corresponding curve, $C:[0, \tau] \longrightarrow \mathcal{P}$. The initial and final states of a curve $\hat{C}$ in $\mathcal{H}_{o}$ that is projected to $C$ in the ray space $\mathcal{P}$ will differ by a phase,

$$
\left|\Psi(\tau)>=e^{i \beta}\right| \Psi(0)>.
$$

This phase $\beta$ can be decomposed into two parts, $\beta=\gamma+\delta$, where $\delta$ is called the dynamical phase which depends on the Hamiltonian,

$$
\delta=-\int_{0}^{\tau}<\Psi \mid H(t) \Psi>d t,
$$


while the remainder, $\gamma$, is the geometrical phase which depends only on the curve $C$ in $\mathcal{P}$. There is a natural $U(1)$ connection defined in $\mathcal{H}_{o}$ such that the holonomy around the path yields the geometric phase $\gamma$. Given $C$ in $\mathcal{P}$, let us define the horizontal lift $C^{\prime}$ in $\mathcal{H}_{o}$, by requiring that $\mid \Phi(t)>$ satisfies,

$$
<\Phi \mid \frac{d}{d t} \Phi>=0 .
$$

Then the state $\mid \Phi(t)>$ acquires a phase when parallel-transported:

$$
\left|\Phi(\tau)>=e^{i \gamma}\right| \Phi(0)>\text {. }
$$

We can define a "connection" $A_{s} \equiv<\Psi(s) \mid \frac{d}{d s} \Psi(s)>$. Under "gauge transformation" $\left|\Psi>\longrightarrow e^{i \lambda(s)}\right| \Psi>, A_{s}$ transforms as $A_{s} \longrightarrow A_{s}+\dot{\lambda}$. Taking $\mid \Psi^{\prime}>$ such that $\left|\Psi^{\prime}(\tau)>=\right| \Psi^{\prime}(0)>$ the geometric phase is

$$
\gamma=i \oint<\Psi^{\prime} \mid \dot{\Psi}^{\prime}>
$$

Berry's phase is recovered when the evolution in $\mathcal{H}_{o}$ is adiabatic and the time dependence of the Hamiltonian is completely contained on external parameters $R_{i}(t)$, such that $R_{i}(\tau)=R_{i}(0)$. The system evolves according to the Schrödinger equation

$$
i \frac{d}{d t}\left|\Psi(t)>=H\left(R_{i}(t)\right)\right| \Psi(t)>.
$$

The adiabaticity is imposed by assuming that an eigenstate $\mid E\left(R_{i}(0)\right)>$ will evolve with $H$ and will be in the state $\mid E\left(R_{i}(t)\right)>$ at time $t$.

The geometric phase is then

$$
\gamma(C)=i \oint_{C}<E\left(R_{i}\right) \mid \nabla_{R} E\left(R_{i}\right)>d R .
$$

In this framework, the Aharonov-Bohm effect can be described as follows [1]. Consider a particle of charge $q$ inside a "perfectly reflecting" box such that the wave function $\Psi_{n}(r)$ is non zero only in the interior of the box. Call $R_{i}$ the vector from the origin of the coordinate system (where the solenoid is located) to the center of the box. When there is no magnetic potential the wave functions have the form $\Psi_{n}(r-R)$ with energies $E_{n}$ independent of $R_{i}$. With non-zero flux the wave functions $<r \mid n\left(R_{i}\right)>$ are obtained by the Dirac phase factor inside the box,

$$
<r \mid n\left(R_{i}\right)>=\exp \left[i q \int_{R}^{r} d r^{\prime} \cdot A\left(r^{\prime}\right)\right] \Psi_{n}(r-R)
$$

Let the box be transported around a circuit $C$ threaded by the flux line. The geometric phase (17) can be then computed using the fact that

$$
\begin{aligned}
<n\left(R_{i}\right) \mid \nabla_{R} n\left(R_{i}\right)> & =\int d^{3} r \Psi_{n}^{*}(r-R)\left[-i q A(R) \Psi_{n}(r-R)+\nabla_{R} \Psi_{n}(r-R)\right] \\
& =-i q A(R),
\end{aligned}
$$


which implies

$$
\gamma(C)=q \oint A(R) \cdot d R=q \phi .
$$

Then, the effect manifests itself as an interference between the particle in the transported box and the one in a box which was not transported round the circuit.

We conclude with two remarks. First note that the solution is single valued and therefore well defined everywhere. Secondly, the factor $e^{i \gamma}$ coincides with the holonomy of the electromagnetic connection, but as we have seen the connection in question is the $U(1)$ connection on the unit sphere $\mathcal{H}_{o}$. This fact will allow us to relate the electromagnetic and gravitational $\mathrm{AB}$ effects.

\section{Space of Solutions}

For a geometric phase as described in the last section to exist, the only conditions that must be satisfied are the existence of a Hilbert Space with a Hermitian inner product and a unitary operator generating time evolution 2 . The existence of such structures in the space-times considered in section 2 is the subject of this section.

In ordinary non-relativistic quantum mechanics, the time evolution of the system is governed by a differential equation which is first order in time, namely the Schödinger equation. The Hamiltonian operator is the infinitesimal generator of time translations and it must be self-adjoint with respect to the Hermitian inner product in order to generate unitary evolution. The Klein-Gordon equation is on the other hand a second order in time equation and it is not clear a-priori that there exist for general spacetimes a well defined "square root" that can be identified with the Hamiltonian.

The Hilbert space structure is however well defined in the case when the underlying space-time is stationary [14] and a Hamiltonian operator can be constructed as the generator of time translations. For completeness, let us recall this construction where the one-particle Hilbert space $\mathcal{H}$ is obtained from the vector space $V$ of real solutions to the Klein-Gordon equation. $\mathcal{H}$ must have the structure of a complex Hilbert space in order to represent quantum states of a particle. For that we need to introduce on $V$ a complex structure $J$ and define, on the complex vector space $(V, J)$, a Hermitian inner product \langle|$>$. Recall that a complex structure $J$ is a linear map $j: V \rightarrow V$ such that $J^{2}=-1$.

The one particle Hilbert space $\mathcal{H}$ would be the Cauchy completion of the complex inner product space $(V, J,<\mid>)$. The complex structure must be compatible with the natural symplectic structure $\Omega$ on $V$;

$$
\Omega\left(\Psi_{1}, \Psi_{2}\right):=\int_{\Sigma} d S^{a}\left(\Psi_{2} \nabla_{a} \Psi_{1}-\Psi_{1} \nabla_{a} \Psi_{2}\right)
$$

\footnotetext{
${ }^{2}$ More general conditions (i.e. non unitary evolution) have been considered in the literature [13], but we will not consider them here.
} 
where $\Sigma$ is any (3-dimensional) Cauchy surface on $\left(M, g^{a b}\right) . J$ is said to be compatible with $\Omega$ iff $\Omega\left(J \Phi_{1}, \Phi_{2}\right)$ is a symmetric, positive definite metric $g(\cdot, \cdot)$ on $V$.

The Hermitian inner product is thus defined,

$$
<\Psi_{1} \mid \Psi_{2}>:=\frac{1}{2} g\left(\Psi_{1}, \Psi_{2}\right)+\frac{1}{2} i \Omega\left(\Psi_{1}, \Psi_{2}\right) .
$$

In the case of stationary space-times the complex structure can be defined from the generator of time evolution $\mathcal{L}_{t}$ on $V\left(\mathcal{L}_{t}\right.$ is a well defined operator in $V$ because it commutes with the Klein-Gordon operator). The complex structure

$$
J:=-\left(-\mathcal{L}_{t} \mathcal{L}_{t}\right)^{-\frac{1}{2}} \cdot \mathcal{L}_{t}
$$

satisfies all the required properties and therefore gives to $(V, J,<\mid>)$ the structure of a complex inner product space. Given a complex structure $J$, one can recover the more familiar language of positive and negative frequency decomposition. In this case the Hilbert Space $\mathcal{H}$ consists of positive frequency solutions $\Psi^{+}$of the Klein-Gordon equation,

$$
\Psi^{+}:=\frac{1}{2}(\Psi-i J \Psi)
$$

and the inner product takes the form,

$$
<\Psi_{1}^{+} \mid \Psi_{2}^{+}>=-i \Omega\left(\overline{\Psi_{1}^{+}}, \Psi_{2}^{+}\right) .
$$

On this space the Schrödinger equation is given by

$$
H \cdot \Psi^{+}=i \hbar \mathcal{L}_{t} \Psi^{+}
$$

This covariant approach (in which one takes real solutions of the KG equation in the full space-time to be the phase space) is completely equivalent to the "canonical" approach in which one considers the phase space to be pairs $(f, p)$ where $f$ is the initial wave function on $\Sigma: f=\left.\Psi\right|_{\Sigma}$, and $p$ is the initial normal derivative: $p=\left.n^{a} \nabla_{a} \Psi\right|_{\Sigma}$.

\section{Geometric Phases in Gravity}

We can now investigate the gravitational Aharonov-Bohm effect by combining the results that were established in the previous sections. In section 2 , we found a solution to the Klein-Gordon equation outside a spinning cosmic string. Let us now confine our quantum system to a box situated at $R_{i}=\left(R_{0}, \theta_{0}, z_{0}\right)$ for $R_{0}>4 \mathrm{~J} /(1-4 \mu)$. This procedure allows us to get rid of two problems: the multivaluedness of the wave function and having to deal with closed timelike curves if.

\footnotetext{
${ }^{3}$ For $R_{0}<\frac{4 J}{(1-4 \mu)}$ the rotational Killing vector field $R^{a}=\left(\frac{\partial}{\partial \phi}\right)^{a}$, which has closed orbits,
} becomes timelike. 
The boundary condition for the wave function inside the box is $\left.\Psi\right|_{\partial \text { box }}=0$. The Hilbert space structure for such solutions can be defined following the procedure outlined in section 4.

Following the electromagnetic case, we proceed by transporting the box round a closed circuit $C$. Since the space-time is axisymetric we can transport the box along orbits of the Killing vector field $R^{a}$. The action of $\mathcal{L}_{R}$ commutes with the KleinGordon operator $\left(\square-m^{2}\right)$. Consequently the action of transporting the box maps the Hilbert space into itself. Therefore we can restrict our analysis to the Hilbert space $\mathcal{H}_{R}$ for the box in the position $R$. Since we are considering the covariant approach, the unitary motion generated by $\mathcal{L}_{R}$ in $\mathcal{H}_{R}$ takes the state $\Psi$ back to the same point in the Hilbert space.

All the framework required to discuss Berry's phase is established. We can now calculate the gravitational geometric phase using (15). We have

$$
\gamma=i \oint_{C}<\Psi\left(R_{i}\right) \mid \mathcal{L}_{R} \Psi\left(R_{i}\right)>
$$

where $\Psi\left(R_{i}\right)$ is given by $(8)$.

The integrand in (27) is evaluated using the Klein-Gordon inner product (25) as follows

$$
\begin{aligned}
<\Psi\left(R_{i}\right) \mid \mathcal{L}_{R} \Psi\left(R_{i}\right)> & =i \int_{\Sigma} d S^{a}\left(\bar{\Psi} \nabla_{a}\left(\mathcal{L}_{R} \Psi\right)-\left(\mathcal{L}_{R} \Psi\right) \nabla_{a} \bar{\Psi}\right) \\
& =-i 4 E J
\end{aligned}
$$

The geometric phase (27) is then

$$
\gamma(C)=8 \pi J E
$$

The effect can be observed by interfering the wave-function in the transported box and another that followed the orbits of the timelike Killing vector field $t^{a}$. We have avoided the region of closed timelike curves by restricting the particle to the box and therefore we do not have problems with the non-Hermiticity of the Hamiltonian [4].

\section{Discussion}

Let's summarize the main results of the paper.

We have found that for those stationary space-times whose Killing vector field has a vanishing twist, solutions to the Klein-Gordon equation can be found using the Dirac phase factor. A geometric phase can thus be found for those space-times given that the existence of a Hermitian inner product in the Hilbert space of solutions is assured. The construction of such phase and its comparison with the electromagnetic $\mathrm{AB}$ effect has been possible because we have interpreted both cases as the holonomy of a $U(1)$ connection on the space of states. The phase depends on the geometry 
of the closed path in the ray space, but will be nonzero when the topology of the underlying space-time is nontrivial. We can say, therefore, that it is of a topological origin. For a spinning cosmic string, we have constructed the phase for a particle going around the string.

We conclude with two remarks. In $2+1$ gravity, the form of the metric (66) for the spinning particle is the general asymptotic form at infinity for "asymptotically flat" space-times. Therefore, the effect is always present near infinity for nontrivial topologies $\left(\Pi_{1}(\Sigma) \neq 0\right)$, when the total angular momentum is non vanishing $(J \neq 0)$. Secondly, our result is a generalization of the geometric phase for a Klein Gordon particle in Minkowski space-time studied in [15].

\section{Acknowledgments}

We would like to thank A. Ashtekar for suggesting this problem. The completion of this work would not have been possible without his advise and extensive discussions. We also thank D. Marolf, M. Varadarajan and J.A. Zapata for discussions. AC was supported by the National University of México (DGAPA-UNAM). MP was supported by CAPES. This work was supported in part by the NSF grant PHY9396246 and by the Eberly research fund of Penn State University.

\section{References}

[1] J.S. Dowker, Nuovo Cimento B52, 129 (1967).

[2] C.J.C. Burges, Phys. Rev. D 32, 504 (1985);

V.B. Bezerra, Phys. Rev. D 35, 2031 (1987);

V.B. Bezerra, J. Math. Phys. 30. 2895 (1989);

V.B. Bezerra, Ann. of Phys. 203, 392 (1990).

[3] A. Ashtekar and A. Magnon, J. Math. Phys. 16, 341 (1975);

J. Anandan, Phys. Rev. D 15, 1448 (1977); 24, 338 (1981);

D. Harari and A.P. Polychonakos, Phys. Rev. D38, 3320 (1988).

[4] P.S. Gerbert and R. Jackiw, Commun. Math. Phys. 124, 229 (1989).

[5] B.S. DeWitt, Phys. Rev. Lett. 16, 1092 (1966).

[6] J. Anandan, Gen. Rel. Grav., in press.

[7] P.O. Mazur, Phys. Rev. Lett. 57, 929 (1986).

[8] S. Deser, R. Jackiw and G. T'Hooft, Ann. of Phys. 152, 220 (1984).

[9] M.A. Peshkin and A. Tonomura, The Aharonov-Bohm effect, Springer Verlag, LNP v. 340 (1989). 
[10] Y. Aharonov and D. Bohm, Phys. Rev. 115, 485 (1959).

[11] M.V. Berry, Proc. R. Soc. Lond. A392, 45 (1984).

[12] Y. Aharonov and J. Anandan, Phys. Rev. Lett. 58, 1593 (1987).

[13] J. Samuel and R. Bandhari, Phys. Rev. Lett. 60, 2339 (1988).

[14] A. Ashtekar and A. Magnon, Proc. R. Soc. Lond. A346, 375 (1975).

[15] J. Anandan and P.O. Mazur, Phys. Lett. A173, 116 (1993). 